\title{
The preablation monocyte/ high density lipoprotein ratio predicts the late recurrence of paroxysmal atrial fibrillation after radiofrequency ablation
}

She-an Chen ${ }^{1,2,3}$, Man-man Zhang ${ }^{1,2}$, Meifang Zheng ${ }^{1,2,3}$, Fei Liu ${ }^{1,2}$, Lei Sun ${ }^{1,2}$, Zheng-yu Bao ${ }^{1,2}$, Fu-kun Chen ${ }^{1,2}$, Hong-xiao $\mathrm{Li}^{1,2}$ and Xiang $\mathrm{Gu}^{1,2^{*}}$ (D)

\begin{abstract}
Background: The monocyte/high-density lipoprotein ratio (MHR) has emerged as a promising alternative biomarker in the fields of cardiovascular disease and atrial fibrillation (AF). This retrospective study was aimed to explore the predictive value of the MHR for the late recurrence of AF after radiofrequency ablation.

Methods: From April 2015 to October 2018, patients with paroxysmal AF who had undergone radiofrequency catheter ablation at Subei People's Hospital of Jiangsu Province were enrolled in our study. All the participants were observed until November 2019 after the procedure. During the postoperative follow up, the patients were categorized into the recurrence group and maintenance of sinus rhythm group based on who had experienced AF recurrence.

Results: One hundred twenty-five patients were diagnosed with paroxysmal AF, with an average age of $61.2 \pm 9.3$ years. Forty-seven patients had developed late recurrence during a mean follow up of $25.1 \pm 12.0$ months. The AF recurrence event rates were significantly increased in the highest MHR tertile compared with those in the lowest MHR tertile (22.0\% vs. 57.1\%; $P<0.05)$. On multivariate logistic regression analysis, the preablation MHR $(O R=1.34 ; 95 \% C l=1.12 \sim 1.60 ; P=$ $0.001)$ and left atrial diameter $(L A D)(O R=1.21,95 \% C l=1.08 \sim 1.35 ; P=0.001)$ were independent risk factors predicting the recurrence of $\mathrm{AF}$ after radiofrequency ablation. Furthermore, receiver operating characteristic $(\mathrm{ROC})$ curve analysis revealed that the area under the curve (AUC) of the MHR was $0.712(95 \% \mathrm{Cl}=0.618 \sim 0.806 ; P=0.000)$ and that of $L A D$ was $0.739(95 \% \mathrm{Cl}=0.653 \sim 0.814 ; P=0.000)$. Z-test found no significant difference between the MHR and LAD regarding the AUC $(Z=0.451 ; P=0.652)$.

Conclusion: An elevated preablation MHR was associated with an increased risk of the postoperative recurrence of AF. Additionally, the MHR independently predicted the late recurrence of paroxysmal AF after radiofrequency ablation, with the same predictive value as LAD.
\end{abstract}

Keywords: Paroxysmal atrial fibrillation, Radiofrequency ablation, Monocyte/HDL ratio, Left atrial diameter, Late recurrence

\footnotetext{
*Correspondence: guxiang@yzu.edu.cn

'Clinical Medical College, Yangzhou University, Yangzhou, Jiangsu, China

${ }^{2}$ Department of Cardiology, Northern Jiangsu People's Hospital, No. 98,

Nantong West Road, Yangzhou 225001, Jiangsu, China

Full list of author information is available at the end of the article
}

(c) The Author(s). 2020 Open Access This article is licensed under a Creative Commons Attribution 4.0 International License, which permits use, sharing, adaptation, distribution and reproduction in any medium or format, as long as you give appropriate credit to the original author(s) and the source, provide a link to the Creative Commons licence, and indicate if changes were made. The images or other third party material in this article are included in the article's Creative Commons licence, unless indicated otherwise in a credit line to the material. If material is not included in the article's Creative Commons licence and your intended use is not permitted by statutory regulation or exceeds the permitted use, you will need to obtain permission directly from the copyright holder. To view a copy of this licence, visit http://creativecommons.org/licenses/by/4.0/ The Creative Commons Public Domain Dedication waiver (http://creativecommons.org/publicdomain/zero/1.0/) applies to the data made available in this article, unless otherwise stated in a credit line to the data. 


\section{Background}

Atrial fibrillation (AF) is a common tachyarrhythmia, accompanied by disorder of atrial electrical activity and mechanical dysfunction. On account of the gradual aging of the population and unhealthy living habits, the global incidence of AF is expected to escalate over the next few decades. During the attack of AF, part of the blood is stagnated in the atrium, and the slow blood flow easily contributes to thrombus formation in the atrium. Therefore, patients with AF are more likely to have a high incidence of stroke and systemic embolism, seriously affecting the quality of life of patients and resulting in a huge burden to public health [1].

Pulmonary vein electrical isolation achieved by radiofrequency catheter ablation has been the cornerstone of catheter-based therapies for AF, with the greatest efficacy as a promising treatment option in patients with paroxysmal AF [2]. The techniques of catheter ablation for AF have undergone a profound evolution recently. Nonetheless, long-term postoperative atrial arrhythmiafree survival remains unsatisfactory, with a high AF recurrence rate of $25-50 \%$ during the postoperative follow up [3]. Therefore, it is of great significance to detect the clinical factors influencing the successful maintenance of the sinus rhythm in patients with AF after ablation.

The monocyte/HDL ratio (MHR), as a new indicator of inflammation and oxidative stress, has been widely explored in the field of cardiovascular disorders, including acute coronary syndrome [4]. Additionally, the MHR was found to be a candidate cardiovascular prognostic marker in chronic kidney disease [5]. Furthermore, the MHR could be used as a predictor of AF recurrence after cryoballoon-based catheter ablation and an elevated preablation MHR was associated with increased recurrence of AF [6]. However, the prognostic value of the MHR in patients with paroxysmal AF after radiofrequency ablation remains controversial. Thus, the study was aimed to investigate the prognostic impact of the MHR on paroxysmal AF after radiofrequency ablation in an observational cohort.

\section{Methods}

\section{Study population}

In this retrospective study, patients with paroxysmal AF who were admitted for pulmonary vein isolation (PVI) treatment using radiofrequency ablation at the Department of Cardiology of Subei People's Hospital of Jiangsu Province from April 2015 to October 2018 were enrolled. All the participants had failed antiarrhythmic drugs previously, and no thrombus was found in the left atrium before surgery. Additionally, patients who had met the following criteria were excluded from the present study: rheumatic heart valve disease, left atrial thrombus, LAD> $55 \mathrm{~mm}$, uncontrolled thyroid dysfunction, congenital heart disease, liver and kidney dysfunction, blood system diseases, contraindication to anticoagulation, and malignant tumors with a life expectancy less than 1 year. The preprocedural informed consent was obtained from all the involved participants, and the study was conducted according to the Helsinki Declaration approved by the ethics committee of Northern Jiangsu People's Hospital.

\section{Catheter ablation procedure and postoperative follow up}

The ablation procedure details had been fully described in the study published in Europace [7]. In our study, under the guidance of the three-dimensional mapping system, Ensite3000 Navx (St. Jude Medical) or CARTO system (Biosense Webster, Inc), electrical isolation of the circumferential pulmonary vein lesions was performed by wellexperienced professors. Additional ablation lines of the left atrium roof should also be implemented in some patients as necessary. The endpoint of the ablation was the absence or dissociation of potentials between the pulmonary vein and left atrium. Additionally, the electrical block of the pulmonary veins was repeatedly evaluated $30 \mathrm{~min}$ after the initial isolation. All the patients took direct oral anticoagulants with warfarin at least 4 weeks before and 3 months after surgery, with a target international normalized ratio of 2.0-3.0. Additionally, all the patients took amiodarone hydrochloride or class IC antiarrhythmic drugs for more than 1 month after ablation. The postoperative follow-up schedules were performed at 3, 6, and 12 months after the surgery and every 6 months thereafter, or whenever they developed discomfort symptoms consistent with recurrent AF. A 48-h Holter electrocardiogram or 12-lead electrocardiogram was recorded at the outpatient follow-up visits. The blanking period was defined as the first 3 months after the AF ablation procedure. Beyond the blank period, AF recurrence referred to the detection of AF/ atrial tachycardia attack with a duration $>30 \mathrm{~s}$, as assessed by electrocardiographic monitoring.

\section{Data collection}

The clinical data of all the patients with AF before ablation were collected and included basic demographic characteristics, disease history, AF duration, the CHA2DS2VASc score, the HATCH score, the monocyte count and other items within the complete blood count, creatinine and uric acid, Cystatin C, D-Dimer, lipid protein profile, left atrial diameter (LAD), left ventricular ejection fraction (LVEF), and follow-up time. The monocyte/HDL ratio (MHR) was calculated as the monocyte count divided by the HDL. All the blood samples were collected $24 \mathrm{~h}$ before the procedure.

\section{Statistical analysis}

All the data were analyzed using SPSS software, version 20.0 for Windows (SPSS Inc., Chicago, Illinois, USA). 
The Kolmogorov-Smirnov criterion was used to assess normality. Continuous variables with a normal distribution were presented as the means \pm standard deviation; otherwise, they were described as medians (interquartile range). The categorical variables were summarized as frequencies and percentages. Comparisons of two continuous variables with a normal distribution were carried out by independent samples Student's t-test, whereas comparisons between two continuous variables were implemented using the Mann-Whitney $U$ test. Categorical variables were compared using chi-squared test or Fisher's exact test. Univariate and multivariate binary logistic regression analyses using the backward likelihood ratio method were employed to determine the risky predictors of AF recurrence. The correlations were assessed using Spearman's rank test. Receiver operating characteristic (ROC) curve analysis was used to assess the predictive value of the risk factors to predict AF recurrence. A two-tailed $P$ value $<0.05$ was considered statistically significant.

\section{Results}

Baseline characteristics and demographical features

One hundred twenty-five patients with paroxysmal AF were enrolled, including 69 males and 56 females, with an average age of $61.2 \pm 9.3$ years. Forty-seven patients had developed late recurrence of AF during a mean follow up of $25.1 \pm 12.0$ months. Depending on the postoperative recurrence of $\mathrm{AF}$, the patients were divided into the recurrence group and maintenance of sinus rhythm group. The baseline demographic characteristics and demographical features, laboratory, and procedural details of both cohorts are summarized in Table 1. The two groups were significantly different in terms of the AF duration, history of diabetes mellitus, body mass index, D-dimer level, monocyte count, HDL cholesterol level, monocyte/HDL ratio, and LAD $(P<0.05)$. Furthermore, the patients were stratified into 3 groups according to the MHR tertile (T1: <5.68; T2: 5.68-8.29; T3: $\geq 8.29$ ) and subgroup analysis showed that patients in T3 had a higher rate of AF recurrence than those in $\mathrm{T} 1$ (22\% vs.

Table 1 Baseline characteristics and demographical features of the study population

\begin{tabular}{|c|c|c|c|}
\hline Variables & sinus rhythm $(n=78)$ & AF recurrence $(n=47)$ & $P$ value \\
\hline Age (years) & $61.5(13.25)$ & $63.0(15.0)$ & 0.717 \\
\hline Gender (female) & $38(48.7 \%)$ & $18(38.3 \%)$ & 0.256 \\
\hline Body mass index $\left(\mathrm{kg} / \mathrm{m}^{2}\right)$ & $24.7 \pm 2.9$ & $26.1 \pm 3.1$ & $0.016^{*}$ \\
\hline Hypertension & $47(60.3 \%)$ & $34(72.3 \%)$ & 0.171 \\
\hline Diabetes mellitus & $9(11.5 \%)$ & $14(29.8 \%)$ & $0.011^{*}$ \\
\hline Coronary artery disease & $19(24.4 \%)$ & $9(19.1 \%)$ & 0.499 \\
\hline Stroke & $13(16.7 \%)$ & $8(17.0 \%)$ & 0.959 \\
\hline AF duration (months) & $12.0(31.25)$ & $24.0(48.0)$ & $0.035^{*}$ \\
\hline Procedure time (minnutes) & $240(60.0)$ & $240(119.0)$ & 0.969 \\
\hline CHA2DS2-VASc score & $2.0(2.0)$ & $2.0(2.0)$ & 0.172 \\
\hline HATCH score & $0.95 \pm 0.89$ & $1.09 \pm 0.93$ & 0.418 \\
\hline Hemoglobin $(\mathrm{g} / \mathrm{L})$ & $137.9 \pm 21.6$ & $137.1 \pm 16.2$ & 0.847 \\
\hline AST (U/L) & $21(10.25)$ & $23(8.0)$ & 0.578 \\
\hline Serum creatinine (umol/L) & $83.0 \pm 18.3$ & $86.6 \pm 19.1$ & 0.301 \\
\hline Uric acid (umol/L) & $309.3 \pm 84.5$ & $325.2 \pm 96.4$ & 0.336 \\
\hline WBC $\left(\times 10^{9} / L\right)$ & $5.40(1.91)$ & $5.69(2.35)$ & 0.359 \\
\hline Monocyte $\left(\times 10^{9} / \mathrm{L}\right)$ & $0.32(0.13)$ & $0.35(0.15)$ & $0.007^{*}$ \\
\hline LDL cholesterol (mmol/L) & $2.42(1.18)$ & $2.36(0.88)$ & 0.521 \\
\hline $\mathrm{HDL}$ cholesterol (mmol/L) & $1.33(0.54)$ & $1.09(0.40)$ & $0.002^{*}$ \\
\hline Monocyte/HDL ratio & $6.34(3.39)$ & $8.29(4.99)$ & $0.000^{*}$ \\
\hline Cystatin C (mg/L) & $0.8(0.39)$ & $0.8(0.30)$ & 0.165 \\
\hline D-Dimer (mg/L) & $0.27(0.21)$ & $0.27(0.14)$ & $0.008^{*}$ \\
\hline $\operatorname{LAD}(\mathrm{mm})$ & $34(6.0)$ & $38(6.0)$ & $0.000^{*}$ \\
\hline LVEF (\%) & $62(1.25)$ & $61(3.0)$ & 0.205 \\
\hline Follow-up duration (months) & $22(20.25)$ & 19 (12.0) & 0.165 \\
\hline
\end{tabular}

${ }^{*} P<0.05$ 
57.1\%; $P<0.05$; Fig. 1). Additionally, subgroup analyses stratified by LAD tertile (T1: < $33.96 \mathrm{~mm}$; T2: 33.96-38 $\mathrm{mm}$; T3: $>38 \mathrm{~mm}$ ) revealed that the rate of recurrence was significantly higher in the highest T3 group than that in the T1 and T2 groups $(P<0.05$; Fig. 1$)$.

\section{Predictors of AF recurrence}

According to univariate binary logistic analysis, AF duration, history of diabetes mellitus, body mass index, monocyte count, the monocyte/HDL ratio, and the LAD were significantly associated with AF recurrence $(P<$ 0.05). However, the CHA2DS2-VASc score, HATCH score, and D-dimer level were identical to those of the sinus rhythm (Table 2). Furthermore, we performed multivariate logistic regression analysis using the backward likelihood ratio method, and the analysis revealed that the $\operatorname{LAD}(O R=1.21 ; 95 \% C I=1.08 \sim 1.35 ; P=0.001)$ and preablation $\mathrm{MHR}(O R=1.34,95 \% \mathrm{CI}=1.12 \sim 1.60$, $P=0.001)$ were independent risk factors predicting the recurrence of AF after radiofrequency ablation. (Fig. 2).

\section{Predictive value of risk factors}

The predictive value of risk factors to predict the recurrence of AF after ablation was evaluated by $\mathrm{ROC}$ analysis. The areas under the curve (AUC) of the MHR and LAD were $0.712(95 \% C I=0.618 \sim 0.806 ; P=0.000)$ and 0.739 (95\% CI $=0.653 \sim 0.814 \quad P=0.000)$, respectively (Fig. 3). Furthermore, Z-test showed no significant difference between the MHR and LAD concerning AUC $(Z=0.451 ; P=0.652)$.

Additionally, correlation analysis revealed a positive correlation of the MHR with the LAD $(r=0.229 ; P=$ 0.01; Fig. 4).

\section{Discussion}

In our retrospective study, we focused more on the relationship between the preprocedural MHR and development of AF recurrence after radiofrequency ablation.

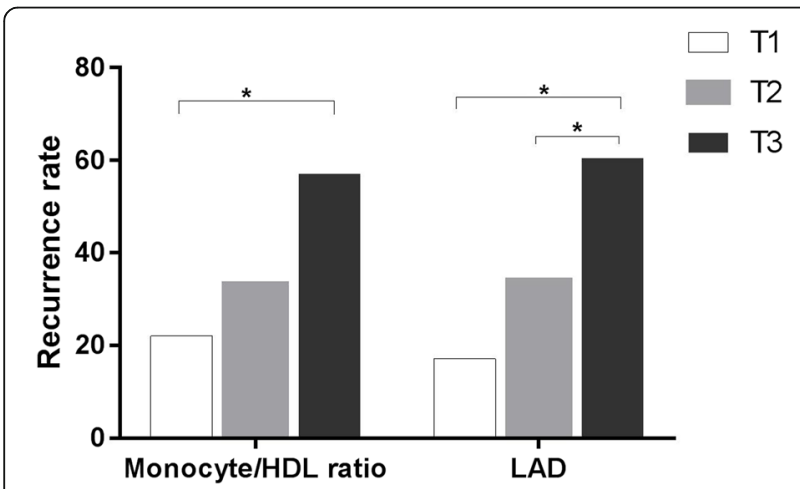

Fig. 1 Percentage of the patients developing post-ablation AF recurrence stratified by tertiles of pre-ablation Monocyte/HDL ratio and LAD
Table 2 Univariate logistic regression modeling results of the AF recurrence

\begin{tabular}{llll}
\hline Variables & \multicolumn{4}{l}{ Univariate model } \\
\cline { 2 - 4 } & OR & $95 \% \mathrm{Cl}$ & $P$ \\
\hline LAD & 1.250 & $1.125-1.389$ & $0.000^{*}$ \\
AF duration & 1.012 & $1.001-1.023$ & $0.026^{*}$ \\
Monocyte & 1.691 & $1.167-2.451$ & $0.005^{*}$ \\
HDL cholesterol & 0.164 & $0.049-0.543$ & $0.003^{*}$ \\
D-Dimer & 4.560 & $1.0-20.794$ & 0.050 \\
Diabetes mellitus & 3.253 & $1.278-8.281$ & $0.013^{*}$ \\
Body mass index & 1.166 & $1.026-1.326$ & $0.019^{*}$ \\
CHA2DS2-VASC score & 1.214 & $0.924-1.597$ & 0.164 \\
HATCH score & 1.180 & $0.793-1.755$ & 0.415 \\
Monocyte/HDL ratio & 1.377 & $1.176-1.614$ & $0.000^{*}$ \\
*P<0.05 & & &
\end{tabular}

The clinical data of 125 patients of paroxysmal AF treated by circumferential pulmonary vein electrical isolation with an average follow-up time of $25.1 \pm 12.0$ months was systematically reviewed. We found that the preablation LAD and MHR were risk factors affecting the therapeutic effect of radiofrequency ablation. Additionally, we had demonstrated that the MHR along with LAD is an independent prognostic factor of AF recurrence in patients with paroxysmal AF who had undergone a single catheter ablation procedure.

AF is the most common atrial arrhythmia in clinical practice, which seriously affects the quality of life of patients and may cause some serious complications, such as heart failure and stroke. Presently, radiofrequency catheter ablation is an effective methods to treat AF. The success rate of the radiofrequency ablation of paroxysmal AF is between 70 and $80 \%$ in 1 year [8] However, some patients will develop relapse of AF after ablation. Many factors, including the AF duration, LAD, and left ventricular dysfunction, have been reported to be related to the recurrence of AF after radiofrequency ablation. The pathophysiological mechanism of AF has not been clearly revealed yet, and many underlying mechanisms participate in the recurrence and maintenance of AF. In recent years, emerging of evidence has revealed that the cross-linked process of inflammation and oxidative stress may result in atrial fibrosis, which is probably the main constituents of AF pathophysiology [9-11] and may play a role in substrate modification by electrical and structural remodeling of the atrium, consequently increasing the susceptibility of AF [12]. Compared with patients with sinus rhythm, the levels of the markers of inflammation and oxidative stress, such as C-reactive protein and interleukin-6, in patients with AF were significantly increased [13]. Inflammation and oxidative stress promote the occurrence of AF, while 


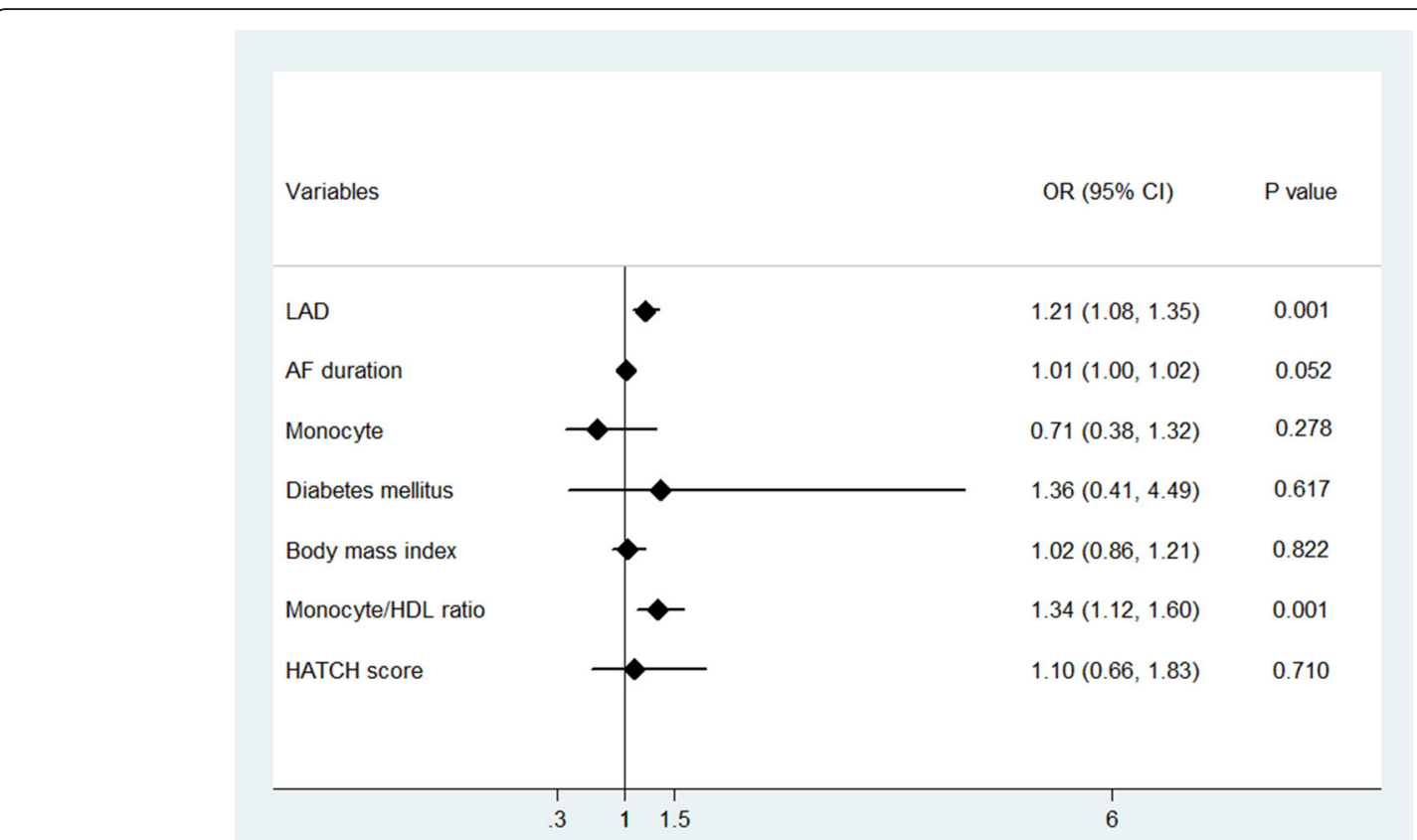

Fig. 2 Forest plot of multivariate logistic regression modeling results of the AF recurrence after ablation

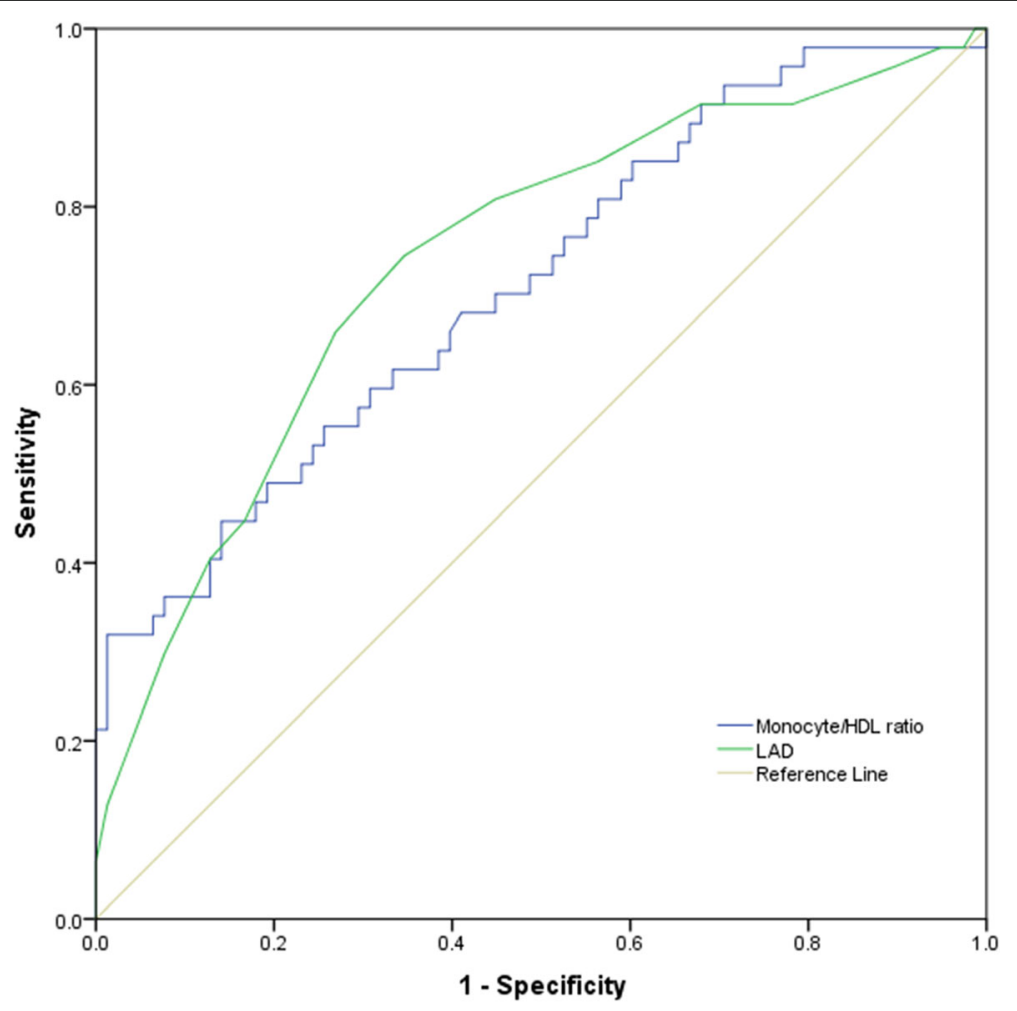

Fig. 3 Receiver operating characteristic curve of pre-ablation variables for predicting AF recurrence after ablation 


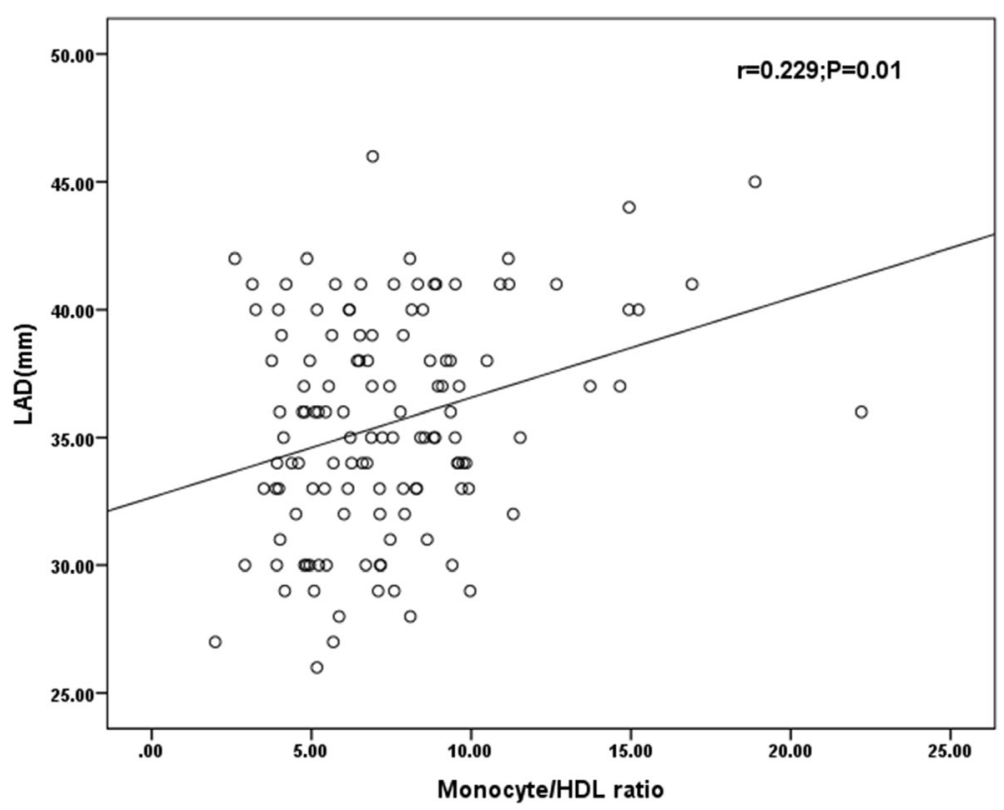

Fig. 4 Correlation of the preablation monocyte/HDL ratio with $\operatorname{LAD}(r=0.229, P=0.01)$

inhibition of the inflammatory response could reduce the risk of AF. A meta-analysis of 50 randomized controlled studies [14] revealed that the prophylactic use of corticosteroids to inhibit the inflammatory response significantly reduced the incidence of postoperative AF in 3323 patients who had undergone cardiac surgery.

As a typical inflammatory cell, monocytes mediate the process of inflammation and oxidative stress by binding to adhesion molecules expressed on damaged vascular endothelial cells and inducing the production of various cytokines as well as play an important role in chronic cardiovascular diseases [15]. However, HDL cholesterol could reduce the expression of CD11b and other adhesion molecules on monocytes and damaged endothelial cells, inhibiting the activation and proliferation of monocytes and thus exerting both anti-inflammatory and antioxidant effects [16]. Recently, the combined circulating monocyte count and serum HDL cholesterol in one fraction, termed 'MHR', was found to be associated with a poor cardiovascular prognosis and emerged as an independent predictor of major cardiovascular events in patients with chronic kidney disease [5]. As a quantitative index of inflammation and oxidative stress, MHR reflects the degree of inflammation and oxidative stress to a certain extent and was found to not only be a predictor of cardiovascular diseases in patients with chronic obstructive pulmonary disease [17] but also to have a certain value in predicting the occurrence of $\mathrm{AF}$ after coronary artery bypass grafting (CABG) [18]. The study included 311 patients who had undergone CABG, and 71 patients developed AF after surgery. The MHR in the group with AF was significantly higher than that of the control group. Additionally, the MHR had also been found as an independent risk factor for AF recurrence after cryoballoon-based catheter ablation. Canpolat et al. [6] enrolled 402 patients who had undergone ablation, and a high MHR before surgery could predict AF recurrence after a mean follow-up time of $20.6 \pm 6.0$ months. In our present study, the increased MHR along with the LAD before radiofrequency ablation is a strong and independent predictor of AF recurrence during the follow up. In clinical practice, we could evaluate the recurrence risk of AF using the preoperative LAD and MHR to select appropriate patients for the procedure, reduce unnecessary interventions and early identify high-risk patients with recurrence, and provide corresponding strengthening intervention measures, to improve the long-term prognosis of the patients. Additionally, the MHR showed the same value of predicting AF recurrence as the LAD, which has been widely accepted as a risk factor for AF recurrence after ablation [19]. Additionally, the MHR was positively correlated with the LAD.

\section{Limitations}

This study is a single-center retrospective study, and the conclusion needs to be further explored by multiple centers. Additionally, the sample size of this study is relatively small, and the postoperative follow-up time is limited. Moreover, the recurrence of asymptomatic AF may be ignored in the follow-up process, contributing to a certain bias in the detection of the postoperative recurrence of AF. 


\section{Conclusion}

In our study, we observed that the elevated preablation MHR was associated with an increased risk of the postoperative recurrence of AF. Additionally, the MHR independently predicted the late recurrence of paroxysmal AF after radiofrequency ablation, with the same predictive value as the LAD.

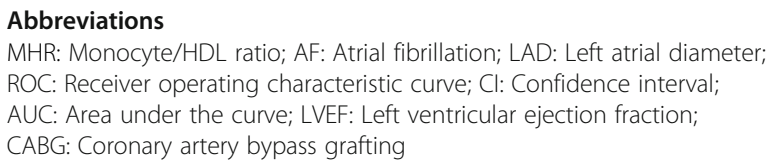

\section{Acknowledgements}

The authors would like to appreciate the assistance of Dr. Ye Zhu, Dr. Yi Zhang, for their valuable advices on the management of the patients. We thank the editor and the reviewers whose comments/suggestions helped improve and clarify this manuscript.

\section{Authors' contributions}

SAC and MMZ: Conceptualization, Investigation, Data curation, Methodology, Formalanalysis, Validation, Writing-original draft, SAC and MMZ contributed equally to this work. MFZ, FL, LS, ZYB, FKC and HXL were involved in gathering the clinical data and editing and revision of the manuscript. XG: Conceptualization, Methodology, Validation, Formal analysis. All the authors were involved in the draft, revision and approval of the final version.

\section{Funding}

This work was supported by funds from science and technology department of Jiangsu province, China (No. BL2013022). Funding is mainly used for chronic follow-up and paper publication.

\section{Availability of data and materials}

The datasets analyzed during the current study are available from the corresponding author on reasonable request.

\section{Ethics approval and consent to participate}

Informed consent were obtained from all involved participants and the study was performed in accordance with the Declaration of Helsinki and approved by the institutionalethical board of Northern Jiangsu Province People's Hospital, Jiangsu Province, China.

\section{Consent for publication}

Not applicable.

\section{Competing interests}

The authors declare that they have no competing interests.

\begin{abstract}
Author details
${ }^{1}$ Clinical Medical College, Yangzhou University, Yangzhou, Jiangsu, China. 2Department of Cardiology, Northern Jiangsu People's Hospital, No. 98, Nantong West Road, Yangzhou 225001, Jiangsu, China. ${ }^{3}$ Clinical Medical College, Dalian Medical University, Dalian 116044, Liaoning, China.
\end{abstract}

Received: 15 June 2020 Accepted: 13 August 2020

Published online: 07 September 2020

\section{References}

1. Pistoia F, Sacco S, Tiseo C, et al. The epidemiology of atrial fibrillation and stroke. Cardiol Clin. 2016;34(2):255-68. https://doi.org/10.1016/j.ccl.2015.12.002.

2. Rottner L, Bellmann B, Lin T, et al. Catheter ablation of atrial fibrillation: state of the art and future perspectives. Cardiol Ther. 2020;9(1):45-58. https://doi. org/10.1007/s40119-019-00158-2.

3. Calkins H, Hindricks G, Cappato R, et al. 2017 HRS/EHRA/ECAS/APHRS/ SOLAECE expert consensus statement on catheter and surgical ablation of atrial fibrillation: executive summary. Europace. 2018;20(1):157-208. https:// doi.org/10.1093/europace/eux275.
4. Acikgoz SK, Acikgoz E, Sensoy B, et al. Monocyte to high-density lipoprotein cholesterol ratio is predictive of in-hospital and five-year mortality in STsegment elevation myocardial infarction. Cardiol J. 2016;23(5):505-12. https://doi.org/10.5603/CJ.a2016.0026.

5. Kanbay M, Solak Y, Unal HU, et al. Monocyte count/HDL cholesterol ratio and cardiovascular events in patients with chronic kidney disease. Int Urol Nephrol. 2014;46(8):1619-25. https://doi.org/10.1007/s11255-014-0730-1.

6. Canpolat U, Aytemir K, Yorgun $\mathrm{H}$, et al. The role of preprocedural monocyte-to-high-density lipoprotein ratio in prediction of atrial fibrillation recurrence after cryoballoon-based catheter ablation. Europace. 2015;17(12): 1807-15. https://doi.org/10.1093/europace/euu291.

7. Efremidis M, Letsas K, Giannopoulos G, et al. Early pulmonary vein reconnection as a predictor of left atrial ablation outcomes for paroxysmal atrial fibrillation. Europace. 2015;17(5):741-6. https://doi.org/10.1093/ europace/euu216.

8. Haegeli LM, Calkins H. Catheter ablation of atrial fibrillation: an update. Eur Heart J. 2014;35(36):2454-9. https://doi.org/10.1093/eurheartj/ehu291.

9. Harada M, Van Wagoner DR, Nattel S. Role of inflammation in atrial fibrillation pathophysiology and management. Circ J. 2015;79(3):495-502. https://doi.org/10.1253/circj.CJ-15-0138.

10. Hu YF, Chen YJ, Lin YJ, et al. Inflammation and the pathogenesis of atrial fibrillation. Nat Rev CardiolNat Rev Cardiol. 2015;12(4):230-43. https://doi. org/10.1038/nrcardio.2015.2.

11. Li J, Solus J, Chen Q, et al. Role of inflammation and oxidative stress in atrial fibrillation. Heart Rhythm. 2010;7(4):438-44. https://doi.org/10.1016/j.hrthm. 2009.12.009.

12. Frustaci A, Chimenti C, Bellocci F, et al. Histological substrate of atrial biopsies in patients with lone atrial fibrillation. Circulation. 1997;96(4):11804. https://doi.org/10.1161/01.cir.96.4.1180.

13. Psychari SN, Apostolou TS, Sinos L, et al. Relation of elevated C-reactive protein and interleukin-6 levels to left atrial size and duration of episodes in patients with atrial fibrillation. Am J Cardiol. 2005;95(6):764-7. https://doi. org/10.1016/j.amjcard.2004.11.032.

14. Ho KM, Tan JA. Benefits and risks of corticosteroid prophylaxis in adult cardiac surgery: a dose-response meta-analysis. Circulation. 2009;119(14): 1853-66. https://doi.org/10.1161/circulationaha.108.848218.

15. Shahid F, Lip GYH, Shantsila E. Role of monocytes in heart failure and atrial fibrillation. J Am Heart Assoc. 2018;7(3):1. https://doi.org/10.1161/jaha.117. 007849

16. Barter PJ, Nicholls S, Rye KA, et al. Antiinflammatory properties of HDL. Circ ResCirc Res. 2004;95(8):764-72. https://doi.org/10.1161/01.res.0000146094. 59640.13

17. Yakar HI, Kanbay A. Could monocyte level/HDL cholesterol ratio predict cardiovascular diseases in patients with COPD? Niger J Clin PractNiger J Clin Pract. 2020;23(4):450-5. https://doi.org/10.4103/njcp.njcp_54_19.

18. Tekkesin Al, Hayiroglu Ml, Zehir R, et al. The use of monocyte to HDL ratio to predict postoperative atrial fibrillation after aortocoronary bypass graft surgery. North Clin Istanb. 2017;4(2):145-50. https://doi.org/10.14744/nci. 2017.53315

19. Liao YC, Liao JN, Lo LW, et al. Left atrial size and left ventricular end-systolic dimension predict the progression of paroxysmal atrial fibrillation after catheter ablation. J Cardiovasc Electrophysiol. 2017;28(1):23-30. https://doi. org/10.1111/jce.13115.

\section{Publisher's Note}

Springer Nature remains neutral with regard to jurisdictional claims in published maps and institutional affiliations.

Ready to submit your research? Choose BMC and benefit from:

- fast, convenient online submission

- thorough peer review by experienced researchers in your field

- rapid publication on acceptance

- support for research data, including large and complex data types

- gold Open Access which fosters wider collaboration and increased citations

- maximum visibility for your research: over $100 \mathrm{M}$ website views per year

At $\mathrm{BMC}$, research is always in progress.

Learn more biomedcentral.com/submission 